\title{
Multifollicular ovary, insulin, and insulin resistance in women with menstrual regularity.
}

\author{
José Alfredo Mendoza Andramuño (EC) Hospital Metropolitano-Quito \\ Wellington Aguirre Solis (EC) Universidad Central Ecuador-Quito \\ Bernardo Aguirre Fernández (EC) Universidad de las Americas-Quito
}

Introduction: Ecosonographic findings of multifollicular ovary (MFO) in young women with menstrual regularity and without stigmata of hyperandrogenism, has not yet a clear explanation, not knowing its connotations in the reproductive function and its relationship with some metabolic parameters as insulinemia, insulin resistance and body weight.

Objectives: Establish the relationship that exists in young women, without stigmata of hyperandrogenism, menstrual regularity, MFO or ecosonographic normal ovaries, insulinemia, insulin resistance and body weight.

Material and methods: We studied a group of 76 young women with MFO and menstrual regularity (A) and another 74 women without MFO and menstrual regularity (B). In all we investigated the levels of glucose, and fasting insulin, insulin resistance (HOMA-IR) and its relation to body weight, we also discard hyperandrogenemia with TTFB (free testosterone).

Results: The mean age was $23.8 \pm 4.8$ years in group $A$ and $24.2 \pm 4.2$ in group $B$. The BMl reached $24.4 \pm 2.8$ and $23.2 \pm 4.3$ in each group respectively, being $>$ to 25 in the $44.7 \%$ of women of the group $A$ and $33.7 \%$ of the group $B(p<0.001)$. Blood glucose levels was $89.9 \pm 8.2 \mathrm{mg} / \mathrm{dl}$ to $85.3 \pm 6.6 \mathrm{mg} / \mathrm{dl}$ in groups $A$ and $B(p<0.001)$. HOMA-IR reached $3.04 \pm 1.4$ in group $A$ and $1.7 \pm 0.6$ in group $B,(p<0.001)$, determining that in group $A$, $46 \%$ had values of HOMA-IR $\geq 3$, while in the $B$, only $4 \%$ exceeded this value $(p<0.0001)$. HOMA weighted by the BMI reached $3.1 \pm 1.4$ in group $A$, and $1.8 \pm 0.6$ in group $B(p<0.001)$. Conclusion: These findings are suggestive that in women with MFO and menstrual regularity, insulin resistance and hyperinsulinemia, would already have an initial deleterious effect on ovarian function, which translates to greater follicular recruitment, with a greater number of antral follicles in the ecosonographic study, which evolution it is still unknown. 